\title{
One year of nuclear cardiology in Europe
}

\author{
Olivier Lairez ${ }^{1,2} \cdot$ Denis Agostini ${ }^{3,4}$
}

Received: 7 July 2016/Accepted: 8 July 2016/Published online: 16 September 2016

(C) The Japanese Society of Nuclear Medicine 2016

$\begin{array}{ll}\begin{array}{l}\text { Abbreviations } \\ 99 \mathrm{~m} \text { Tc }\end{array} & \begin{array}{l}99 \mathrm{~m}-\text { technetium } \\ \text { BMIPP }\end{array} \\ & \begin{array}{l}\text { Iodine-123- } \beta \text {-methyl iodo-phenyl-pentadecanoic } \\ \text { acid }\end{array} \\ \text { CAD } & \text { Coronary artery disease } \\ \text { CRT } & \text { Cardiac resynchronization therapy } \\ \text { CZT } & \text { Cadmium zinc telluride } \\ \text { ED-B } & \text { Extra-domain B of fibronectin } \\ \text { MPI } & \text { Myocardial perfusion imaging } \\ \text { SPECT } & \text { Single-photon emission computed tomography }\end{array}$

\section{Preamble}

Since the introduction of the need to use imaging for the monitoring of cardiomyopathies and particularly ischemic cardiomyopathies in international guidelines, articles reporting the place, the accuracy and the prognostic impact of cardiac imaging have grown exponentially. During 2015, the European Journal of Nuclear Medicine and Molecular Imaging published selected pre-clinical, technical and clinical studies sweeping the most advanced items in nuclear cardiology. Advances in nuclear cardiology led the Journal to publish revision from the European

Denis Agostini

agostini-de@chu-caen.fr

1 Department of Nuclear Medicine, Rangueil University Hospital, Toulouse, France

2 Cardiac Imaging Center, Toulouse University Hospital, Toulouse, France

3 Department of Nuclear Medicine, Caen University Hospital, Caen, France

4 Normandy University, EA 4650 Caen, France
Association of Nuclear Medicine guidelines for radionuclide myocardial perfusion imaging (MPI) [1]. The current review provides a summary of selected articles representative of the most interesting advances in the field of nuclear medicine published in the Journal during 2015.

\section{Development of hardwares and impact on practices for myocardial perfusion imaging}

\section{Context}

Last years had seen growing interest in reducing acquisition times for MPI. This dynamics has led to the development of new hardware capable of acquiring MPI studies in a fraction of the time usually required by conventional tools, with the same image quality and diagnosis accuracy. The two most significant advances in this field were represented by the development of cadmium zinc telluride (CZT) crystals and multifocal collimation by IQ-SPECT. CZT crystals had opened the door for dynamic SPECT leading to the exciting perspectives of myocardial blood flow and coronary reserve quantification in a daily practice. Meanwhile, IQ-SPECT, without allowing the same perspectives for dynamic SPECT, can reduce acquisition times leading to improve patient throughput, facilitate the workload of department of nuclear medicine and, by extension, reduce radiation exposure. IQ-SPECT, which can be applied as a plug-in on general-purpose cameras, is a good compromise for centers with general activity that do not want to make the investment of dedicated cardiac camera.

Considering the emergence of these new cameras, the article of Caobelli et al. drew our attention by reporting the feasibility of one-eighth time gated myocardial perfusion SPECT using IQ-SPECT in clinical practice [2]. 


\section{Method and results}

Caobelli and co-authors [2] hypothesized that images obtained by acquisition times one-eighth of standard times would be statistically equivalent to the standard protocol. They compared global and regional perfusion defects, images quality scores, left ventricular volumes and function obtained from set of images acquired at $6 \mathrm{~s} / \mathrm{view}$ and $12 \mathrm{~s} / \mathrm{view}$ in 50 patients. They found a significant decrease in image quality with the $6 \mathrm{~s} /$ view compared with the $12 \mathrm{~s} /$ view. But all images were rated as adequate and the difference was considered as clinically negligible. They did not find difference between set of images for perfusion scores, leading to a similar image-based diagnosis of global and regional coronary artery disease (CAD). A similar pattern was observed for semi-quantitative analysis using summed scores. Left ventricular volumes were higher in images obtained with the shorter acquisition time than with the longer acquisition time, but left ventricular ejection fraction did not significantly differ between the two acquisition times. This study supported the use of a shorter acquisition time in clinical practice (from 20 to $2.5 \mathrm{~min}$ ), and by extension, patient radiation exposure reduction using IQ-SPECT for MPI.

\section{The use of hybrid cameras for attenuation correction}

\section{Context}

Like PET few years ago, and because specificity of MPI cans be limited by attenuation artifacts, nowadays, most of constructor offer to correct attenuation using computed $\mathrm{X}$-ray tomography to acquire linear attenuation coefficient maps. However, several reports on the use of attenuation correction in MPI have shown discordant results and its utility in routine clinical practice is still debated. Until now, analysis of wall thickening using ECG gating has been routinely used to identify attenuation artifacts. Considering these two different approaches, Benkiran et al. offer to evaluate the consequences of computed X-ray tomography-based attenuation correction and wall thickening analysis on the diagnostic performance of MPI in routine clinical settings [3].

\section{Method and results}

Benkiran and co-authors [3] explored prospectively, in 70 patients, the relative impact of computed tomography-based attenuation correction and the segmental thickening scores using ECG-gating on the sensitivity, specificity, and overall accuracy on the basis of coronary angiography (for $19 \%$ of patients) and ECG, echocardiography, and clinical followup (for $81 \%$ of patients). In their population, the prevalence of ischemic heart disease was low with $81 \%$ of patients considered free of CAD; and 3,11, and $4 \%$ of patients with myocardial ischemia, myocardial necrosis, and both ischemia and necrosis, respectively. The sensitivity, specificity, and overall accuracy were, respectively, 77, 60, and $63 \%$ for 'standard' SPECT analysis (without attenuation correction or wall thickening analysis); 67,81 , and $79 \%$ for computed tomography-based attenuation correction analysis; and 69, 98, and $93 \%$ for segmental thickening-based analysis. Differences were statistically significant for specificity and overall accuracy by taking 'standard' SPECT analysis as reference. Interestingly, segmental thickening-based analysis led to 33 and $1 \%$ of right and wrong change, respectively, in the final interpretation, whereas CT-based attenuation correction analysis led to 24 and $13 \%$ of right and wrong change, respectively, in the final interpretation. The additional effective dose for computed tomography-based attenuation correction was $2 \mathrm{mSv}$. Although that both computed tomography-based attenuation correction analysis and segmental thickening-based analysis led to improved specificity and diagnostic accuracy compared with 'standard' SPECT analysis, all these results led the authors to consider gated SPECT as a self-sufficient modality for CAD screening and follow-up; and to consider attenuation correction only when doubt remains after gated SPECT analysis.

\section{Looking beyond myocardial perfusion}

\section{Context}

Because balanced multivessel disease can result in falsely normal myocardial perfusion imaging study, new tools are needed to increase the accuracy of SPECT for the assessment of CAD.

As highlight in the editorial of Ben-Haim and Agostini [4], CZT crystals provide improved sensitivity (eight to ten times better than conventional SPECT), spatial resolution and energy resolution. These improvements, in addition to allowing dynamic SPECT with kinetic analysis of myocardial tracer uptake, provide incremental diagnostic information over perfusion data alone. Myocardial ischemia results, generally, in sequential alterations in myocardial diastolic, then systolic functions followed by electrographic and symptomatic manifestations. Based on the capacity of CZT cameras to better study myocardial behavior, Gimelli et al. evaluated the interplay between myocardial ischemic burden and post-stress left ventricular diastolic and systolic abnormalities in patients with suspected or known CAD [5]. 
On the other hand, the emergence of hybrid scanners combining SPECT and CT, allowing combination of functional and morphological approach, opens new perspectives for coronary imaging. Because high coronary artery calcium score is associated with a greater degree of stenosis and ischemia, combination of coronary artery calcium score and myocardial perfusion assessment could allow detecting falsely normal myocardial perfusion SPECT in patients with balanced multivessel disease. Yuoness et al. explored the incidence of severe CAD in stable symptomatic patients with normal myocardial perfusion imaging and high coronary artery calcium score [6].

\section{Method and results}

Gimelli and co-authors explored the relationships among CAD severity and extent, regional myocardial ischemia and early post-stress left ventricular functional performance in 471 consecutive patients with angina-like chest pain and known or suspected CAD referred for CZT myocardial perfusion scintigraphy [5]. They found that the magnitude of post-stress left ventricular systolic dysfunction paralleled the increase in coronary artery extent, whereas the occurrence of a significant stress-induced systolic dysfunction was limited to patients with multivessel disease. Finally, summed difference score and stress-to-rest peak filling rate ratio were independent predictors of significant CAD surpassing the effects of measures of stress-induced volumetric overload and systolic impairment. Experimental studies have suggested that stress-induced left ventricular diastolic stunning is an earlier and more durable manifestation of ischemia than systolic stunning [7]. This study shows how myocardial perfusion imaging by CZT scintigraphy, allowing early assessment of stress-induced left ventricular abnormalities, offers the opportunity to combine the evaluation of myocardial perfusion with left ventricular diastolic and systolic functions, to better cover the "ischemic cascade".

For their part, Yuoness and co-authors explored 26 consecutive symptomatic patients without known CAD with normal perfusion myocardial SPECT (even for left ventricular systolic function) and a coronary artery calcium score of $>1,000$ [6]. All patients underwent invasive coronary angiography, which found $58 \%$ of severe CAD. Among patients with severe CAD, $60 \%$ had three-vessel disease, $47 \%$ required percutaneous coronary intervention and $54 \%$ underwent coronary artery bypass grafting. This study opens a door for hybrid scanners in the evaluation of coronary artery atherosclerosis associating functional assessment by myocardial perfusion imaging and morphological assessment by coronary artery calcium score to overcome false-negative studies in patients with multivessel disease.

\section{Integrating exercise tolerance to better assess prognosis}

\section{Context}

SPECT myocardial perfusion imaging is routinely used for risk assessment in patients with known or suspected CAD. It provides additional prognostic information on clinical data, coronary anatomy and exercise stress score. Marini et al. evaluated the prognostic value of combining imaging-derived indexes of ischemia and exercise tolerance [8].

\section{Method and results}

Marini and co-authors combined imaging indexes of ischemia severity with maximal achieved workload and maximal rate pressure product in 1,502 patients with rest and exercise gated SPECT myocardial perfusion imaging [8]. Clinical prognostic index was defined by summed difference score divided by maximal rate pressure product* $10^{5}$. Among the 357 patients with reversible perfusion defects, after multivariate analysis, summed difference score showed a weak predictive value for major adverse cardiac events. Normalization of ischemic threshold improved summed difference score risk stratification and clinical prognostic index correctly predicted the incidence of major adverse cardiac events both in univariate and multivariate analysis. This study confirms that normalizing imaging score of ischemia severity and extension by clinical indexes of exercise tolerance improves risk stratification for major cardiac events. These results supported the integration of the clinical evaluation of ischemic threshold as an easily applicable tool in the interpretation of SPECT myocardial perfusion imaging.

\section{Can nuclear cardiology help to predict response to cardiac resynchronization therapy?}

\section{Context}

Conventional criteria for selection of patients for cardiac resynchronization therapy (CRT) lead to about one-third of no significant benefits [9]. Although mechanical cardiac dyssynchrony has been recognized as an essential criterion for response to CRT, conventional imaging tests for dyssynchrony have not yet been shown to be of value in selecting patients for CRT. This had led several authors to question the value of nuclear cardiology imaging to assess the left ventricular dyssynchrony and predict CRT response. 


\section{Method and results}

Mukherjee and co-authors evaluated prospectively gated myocardial perfusion SPECT in the prediction of response to CRT in 32 non-ischemic dilated cardiomyopathy patients [10]. First-harmonic fast Fourier transform was used to extract a phase array from MPI at baseline and 3 months after CRT implantation. They showed that responders had significantly larger phase standard deviation and phase histogram bandwidth compared with nonresponders, based on NYHA functional class improvement and increase in left ventricular ejection fraction by more than $5 \%$ on follow-up. Receiver operating characteristic curve analysis demonstrated $86 \%$ sensitivity and $80 \%$ specificity at a cutoff value of $43^{\circ}$ for phase standard deviation; and $86 \%$ sensitivity and $80 \%$ specificity at a cutoff value of $128^{\circ}$ for phase histogram bandwidth in the prediction of response to CRT.

For their part, Chen and co-authors evaluated prospectively equilibrium radionuclide angiography in the prediction of response to CRT in 24 heart failure patients (79\% ischemic and $21 \%$ idiopathic) [11]. Left ventricular phase shift and left ventricular phase standard deviation were obtained on the phase image and used to predict response to CRT based on a reduction of at least $15 \%$ in left ventricular end-systolic volume $48 \mathrm{~h}$ after CRT implantation. Baseline left ventricular phase shift and left ventricular phase standard deviation were larger in responders. Responders demonstrated a significant reduction of left ventricular phase shift and left ventricular phase standard deviation $48 \mathrm{~h}$ after CRT implantation. Receiver operating characteristic curve analysis demonstrated $80 \%$ sensitivity and $89 \%$ specificity at a cutoff value of $25 \%$ for left ventricular phase shift; and $87 \%$ sensitivity and $89 \%$ specificity at a cutoff value of $8.5 \%$ for left ventricular phase standard deviation in the prediction of response to CRT.

All these results suggest that left ventricular phase analysis derived from both myocardial perfusion SPECT and equilibrium radionuclide angiography could be useful for prediction of acute and late response to CRT.

\section{The new era of PET/MRI in clinical practice}

\section{Context}

The recent emergence of hybrid PET/MRI has opened new perspectives for cardiovascular applications. PET and MRI provide additional and complementary information. Numerous recent studies suggest promising applications in the cardiovascular field [12]. As such, the article of Nappi et al. is a good example of the potential of hybrid scanners in clinical practice [13].

\section{Method and results}

Nappi and co-authors investigated the potential role of PET/MR imaging in the assessment of cardiac involvement in 13 patients with Anderson-Fabry disease without cardiac symptoms [13]. They performed coregistration of myocardial ${ }^{18}$ F-FDG uptake by PET and MR images including cine-MRI, T1- and T2-weighted, and late gadolinium enhancement images. They found $38 \%$ of left ventricular hypertrophy and focal late gadolinium enhancement. Among these patients, $60 \%$ had positive T2-weighted images and focal FDG uptake in the corresponding myocardial territory. The $40 \%$ remaining patients had negative T2-weighted images but heterogeneous FDG uptake (assessed by measure of the coefficient of variation of the uptake of the 17 segments). All these patients had elevated cardiac troponin values. Among the patients without left ventricular hypertrophy or late gadolinium enhancement, $29 \%$ had heterogeneous myocardial FDG uptake. These patients were hemizygous for the mutation and started enzyme replacement therapy long time ago. This study illustrates the capacity of new hybrid scanners integrating PET and MRI to bring morphological, functional and tissue information for the assessment of pro-inflammatory and fibrotic myocardial diseases.

\section{The emergence of molecular imaging}

\section{Context}

Numerous metabolic factors can impact cardiovascular prognosis. One of the most striking examples is the impact of chronic kidney on cardiovascular prognosis. Kidney disease induces activation of growth factors, proto-oncogenes, plasma norepinephrine, cytokines, and angiotensin II. These factors regulate intracellular processes that accelerate cardiac hypertrophy, myocardial fibrosis, and apoptosis [14]. The common point of all these pathologic processes is the impairment in myocardial fatty acid metabolism. This impaired metabolism can now be explored by imaging, and the new perspectives brought by the recent works of Nishimura et al. [15] drew our attention.

\section{Method and results}

Nishimura and co-authors explored myocardial fatty acid metabolism by SPECT using the iodinated fatty acid analogue, iodine-123- $\beta$-methyl iodo-phenyl-pentadecanoic acid (BMIPP) on 155 hemodialysis patients without CAD (based on the results of invasive coronary 
angiography) [15]. Tracer uptake of each segment was visually graded and assigned an uptake score of 0 (normal), 1 (mildly reduced), 2 (moderately reduced), 3 (severely reduced), or 4 (none), and the BMIPP score was calculated by summing the results of the 17 myocardial segments. Focal pattern of tracer uptake was defined as BMIPP defect scores $\geq 2$ in $\geq 2$ consecutive segments. Among the 155 included patients, 95 patients had abnormal BMIPP SPECT findings (defined by BMIPP score $\geq 6$ ) and were enrolled in the study. Focal pattern in BMIPP SPECT was found in $44 \%$ of patients. After a follow-up of $5 \pm 2$ years, cardiac deaths were higher in the focal $(71 \%)$ than in the non-focal $(23 \%$, $P<0.001)$ group. After multivariate analysis, BMIPP summed score and focal pattern were independent risk factors for cardiac with hazard ratios of 8.7 and 2.3, respectively. Among patients with focal pattern, $48 \%$ showed focally reduced uptake of BMIPP in the single coronary artery area. The predictive potential for coronary territorial BMIPP scores for cardiac deaths was higher in the left anterior descending artery area. These findings show that severe impairment in BMIPP SPECT and focally impaired myocardial fatty acid imaging in the left anterior descending artery area are strong risk factors for cardiac death in hemodialysis patients. Over the results applied to hemodialysis patients, the study illustrates to potential of BMIPP SPECT to explore myocardial metabolism and opens the door of molecular imaging in the field of cardiac imaging.

\section{Identifying critical molecular mechanisms of atherosclerotic disease to develop novel therapeutic strategies}

\section{Context}

High-risk atherosclerotic plaque represents the susceptibility of a plaque to rupture, and its detection has become the goal of new imaging techniques. The morphological approach does not provide information regarding functional variables, such as plaque metabolism and inflammation, which are recognized markers of vulnerability. Molecular imaging techniques can quantify specific biological processes, thus giving insight into the functional parameters of tissues [16]. The extra-domain B (ED-B) of fibronectin was found recently to be expressed in advanced atherosclerotic plaques. This led Dietrich et al. to test whether ED-B antibody conjugated with 99 m-technetium $\left({ }^{99 \mathrm{~m}} \mathrm{Tc}\right)$ would be suitable for in vivo visualization of atherosclerotic plaque lesions in atherosclerotic westernfed diet Apo-E-/- mice [17].

\section{Method and results}

Seven Apo-E-/- mice fed with high-cholesterol diet were imaged with high-resolution MRI $24 \mathrm{~h}$ prior to ${ }^{99 \mathrm{~m}} \mathrm{Tc}$ labeled ED-B antibody SPECT imaging and compared with 5 wild-type mice fed on normal chow used as control. In vivo ${ }^{99 \mathrm{~m}}$ Tc-labeled ED-B antibody SPECT imaging of the high-fat diet Apo E-/- mice demonstrated a significant higher signal activity than controls in the thoracic area, which co-localized with aortic arch and the supraaortic arteries. The specificity of this increased signal activity was confirmed by ex vivo autoradiography. By histology, high-fat diet Apo E-/- mice expressed highrisk atherosclerotic plaques with inflammatory processes characterized by infiltrating macrophages and lymphocytes as compared with controls. There was a significant correlation between the size of aortic plaque areas identified by ${ }^{99 m}$ Tc-labeled ED-B antibody autoradiography and the macrophage-infiltrated plaque areas by histology. These promising results illustrate the potential of molecular imaging, and particularly of nuclear imaging, in the field of vulnerable plaque identification.

\section{Conclusion}

Nuclear cardiology in Europe during 2015 was representative of the evolutionary turning point that knows the nuclear cardiology: the development of faster cameras, which change our daily practice and open the way for dynamic SPECT, the surpassing of myocardial perfusion, which improve myocardial assessment and prognosis, the emergence of hybrid imaging, that change our way of understanding the disease and the perspective for new tracers, which opens the way of molecular imaging. All these points bring exciting perspectives for the next years.

\section{References}

1. Verberne HJ, Acampa W, Anagnostopoulos C, et al. EANM procedural guidelines for radionuclide myocardial perfusion imaging with SPECT and SPECT/CT: 2015 revision. Eur J Nucl Med Mol Imaging. 2015;42:1929-40.

2. Caobelli F, Thackeray JT, Soffientini A, Bengel FM, Pizzocaro C, Guerra UP. Feasibility of one-eighth time gated myocardial perfusion SPECT functional imaging using IQ-SPECT. Eur J Nucl Med Mol Imaging. 2015;42:1920-8.

3. Benkiran M, Mariano-Goulart D, Bourdon A, Sibille L, Bouallegue FB. Is computed tomography attenuation correction more efficient than gated single photon emission computed tomography analysis in improving the diagnostic performance of myocardial perfusion imaging in patients with low prevalence of ischemic heart disease? Nucl Med Commun. 2015;36:69-77. 
4. Ben-Haim S, Agostini D. Dynamic SPECT: evolution of a widely available tool for the assessment of coronary flow reserve. Eur $\mathbf{J}$ Nucl Med Mol Imaging. 2015;42:302-4.

5. Gimelli A, Liga R, Giorgetti A, Kusch A, Pasanisi EM, Marzullo P. Relationships between myocardial perfusion abnormalities and poststress left ventricular functional impairment on cadmiumzinc-telluride imaging. Eur $\mathrm{J}$ Nucl Med Mol Imaging. 2015;42:994-1003.

6. Yuoness SA, Goha AM, Romsa JG, et al. Very high coronary artery calcium score with normal myocardial perfusion SPECT imaging is associated with a moderate incidence of severe coronary artery disease. Eur $\mathrm{J}$ Nucl Med Mol Imaging. 2015;42:1542-50.

7. Ishii K, Suyama T, Imai M, et al. Abnormal regional left ventricular systolic and diastolic function in patients with coronary artery disease undergoing percutaneous coronary intervention: clinical significance of post-ischemic diastolic stunning. J Am Coll Cardiol. 2009;54:1589-97.

8. Marini C, Acampa W, Bauckneht M, et al. Added prognostic value of ischaemic threshold in radionuclide myocardial perfusion imaging: a common-sense integration of exercise tolerance and ischaemia severity. Eur $\mathbf{J}$ Nucl Med Mol Imaging. 2015;42:750-60.

9. Abraham WT, Fisher WG, Smith AL, et al. Cardiac resynchronization in chronic heart failure. N Engl J Med. 2002;346:1845-53.

10. Mukherjee A, Patel CD, Naik N, Sharma G, Roy A. Quantitative assessment of cardiac mechanical dyssynchrony and prediction of response to cardiac resynchronization therapy in patients with nonischaemic dilated cardiomyopathy using gated myocardial perfusion SPECT. Nucl Med Commun. 2015;36:494-501.

11. Chen Y, Yan J, Zhao S, Long Q, Wang H, Wang L. Efficacy of equilibrium radionuclide angiography to predict acute response to cardiac resynchronization therapy in patients with heart failure. Nucl Med Commun. 2015;36:610-8.

12. Ratib O, Nkoulou R. Potential applications of PET/MR imaging in Cardiology. J Nucl Med. 2014;55:40S-6S.

13. Nappi $\mathrm{C}$, Altiero $\mathrm{M}$, Imbriaco $\mathrm{M}$, et al. First experience of simultaneous PET/MRI for the early detection of cardiac involvement in patients with Anderson-Fabry disease. Eur J Nucl Med Mol Imaging. 2015;42:1025-31.

14. Amann K, Kronenberg G, Gehlen F, et al. Cardiac remodelling in experimental renal failure-an immunohistochemical study. Nephrol Dial Transplant. 1998;13:1958-66.

15. Nishimura M, Hashimoto T, Tamaki N, Kobayashi H, Ono T. Focal impairment in myocardial fatty acid imaging in the left anterior descending artery area, a strong predictor for cardiac death in hemodialysis patients without obstructive coronary artery disease. Eur J Nucl Med Mol Imaging. 2015;42:1612-21.

16. Lairez O, Fayad ZA. Imaging of atherosclerosis: can molecular imaging do more? Arch Cardiovasc Dis. 2013;106:551-3.

17. Dietrich T, Berndorff D, Heinrich T, et al. Targeted ED-B fibronectin SPECT in vivo imaging in experimental atherosclerosis. Q J Nucl Med Mol Imaging. 2015;59:228-37. 